\title{
INVESTIGACIONES
}

\section{FORMACION PERMANENTE DE PROFESORES: ¿QUIEN ES EL FORMADOR DE FORMADORES?**}

Teacher training: who is the teacher trainer?

\author{
Christian Miranda Jaña, Pablo Rivera Rivera** \\ Instituto de Filosofía y Estudios Educacionales, Universidad Austral de Chile, \\ Campus Isla Teja, Casilla 567, Valdivia, Chile. \\ christianmiranda@uach,cl
}

\section{Resumen}

El artículo se propone describir el perfil profesional de los formadores de formadores de los Programas de Postítulo para segundo ciclo básico desde una perspectiva de género. A partir de una metodología cuantitativa de diseño descriptivo-censal $(\mathrm{N}=363)$, con uso de estadística descriptiva en el análisis, el estudio presenta como resultados la caracterización del formador en términos personales, académicos y de desempeño científico, lo cual es discutido a la luz del debate actual sobre la enseñanza eficaz y el rol del género en el desarrollo profesional docente.

Palabras clave: formación permanente, formador de formadores, perfil profesional docente, programa de postítulo, género.

\begin{abstract}
The article sets out to describe the Professional Profile of Trainers of Teachers who took part in postgraduate courses for primary school, from a gender perspective. In addition, it intends to contribute to the debate of professional development from a key actor, who is not usually considered when educational policies are designed: the teacher trainer. From a quantitative descriptive methodology of censal character $(\mathrm{N}=363)$, the study presents as a result the characterization of a profile that identifies personal as well as academic characteristics and performance of scientific productivity of the trainers. This is discussed at the light of the current debate on the effective education and the role of the gender in the teachers professional development.
\end{abstract}

Key words: professional development, trainers of teachers, educational professional profile, postgraduate program.

\footnotetext{
* $\quad$ Este estudio forma parte del estudio ¿Qué hace a la formación permanente de profesores eficaz?, financiado por CONICYT a través del proyecto FONDECYT N ${ }^{\circ} 11060128$.

** Estudiante de doctorado en Ciencias Humanas, Universidad Austral de Chile, Becario de CONICYT.
} 


\section{INTRODUCCION}

El presente artículo emerge desde la reflexión de los resultados obtenidos en una línea de investigación, cuya interrogante general es qué hace a la formación permanente de profesores eficaz. Esta se operacionalizó en preguntas orientadas a develar quién es el formador de formadores (FF) desde una perspectiva de género. En tal sentido, lo que sigue introduce dos temas poco abordados e interrelacionados por la literatura especializada sobre el desarrollo profesional de profesores en general y los Programas de Postítulo (PP), en lo particular: género y FF.

Los Programas de Postítulo se crean como una respuesta a las recomendaciones de la OCDE (2004) respecto de la inseguridad de los docentes de segundo ciclo básico a la hora de enseñar los contenidos disciplinares. Los PP se diseñan ese mismo año como un esfuerzo conjunto entre el Ministerio de Educación (MINEDUC) y universidades que forman profesores (Plan piloto). La propuesta es liderada por el Centro de Perfeccionamiento, Experimentación e Investigación Pedagógica (CPEIP) y encauzada en el Polo de Actualización Curricular (Miranda et al. 2007). Se implementa el plan en cuatro instituciones del país ${ }^{1}$, siendo los primeros Programas de Postítulo en Matemáticas (PPMAT) y Estudio y Comprensión de la Naturaleza (PPECN).

A partir del 2006, y tras una primera evaluación satisfactoria (Cancino 2005), se invita al resto de universidades a proponer programas en las áreas antes mencionadas, agregando Estudio y Comprensión de la Sociedad (PPECS) y Lenguaje y Comunicación (PPLC). Al año 2007, los postítulos impartidos fueron aproximadamente cuarenta y las universidades participantes diecisiete. Desde el año 2008 los PP expanden su oferta a la totalidad de la educación básica, en iguales áreas curriculares.

En la actualidad existe un creciente interés por evaluar la eficacia de estos programas. Ejemplo de ello son la investigación realizada por González y Trebilcock (2007) cuyo énfasis estuvo en describir la relación entre la interacción pedagógica y la apropiación de contenidos (PPMAT y PPLC) y el estudio de Miranda (2007) que centró su análisis en los factores que inciden en el impacto de los Postítulos (PPMAT y PPECN) en el conocimiento, la práctica y aprendizaje estudiantil. Finalmente Fritz et al. (2008) realizan un estudio que enfatiza en la presencia de la perspectiva de género en los PP desde el punto de vista de su presencia o ausencia curricular. Si bien éstos abordan el problema de la eficacia de los programas, poco se avanza en la comprensión del rol de formadores de formadores, no solo referido a su impacto en el aprendizaje docente sino en su caracterización personal y profesional, pues tal como lo plantean Loewenberg, Simons y Wu (2008) los formadores son cruciales en las oportunidades de los estudiantes de aprender, y diferencias sustanciales del logro de estudiantes son atribuibles a diferencias entre los profesores, siendo uno de ellos su género.

El Programa de las Naciones Unidas para el Desarrollo (PNUD) diseñó un indicador sobre discriminación de género que evalúa la igualdad de éste en las esferas de participación política y económica (UNICEF 2006). De la información oficial presentada por el PNUD se concluye que el patrón de desarrollo humano es inequitativo, ante lo cual instaló la equidad de género como objetivo prioritario en la Agenda del Milenio de las Naciones Unidas, donde sugiere la implementación de políticas que compensen la

1 U. de La Serena, U. de Santiago de Chile. U. de Concepción, U. Católica de Temuco. 
desigualdad de trayectorias y no sólo de oportunidades para hombres y mujeres (Jewkes 2004). En este sentido, la educación y actores involucrados son identificados como factores clave para incidir simultáneamente sobre la equidad, el desarrollo integral y el ejercicio de ciudadanía plena (Stacki et al. 2003; Magendzo 2003).

Efectivamente, la evidencia científica es consistente en afirmar que entre los aspectos intrainstituciones más relevantes en la explicación de la calidad educativa están las características socioeconómicas (Ej., género), institucionales (Ej., equipo de docentes) y pedagógicas (Ej., perfil profesional) (OCDE 2008).

En este documento se presentan los presupuestos conceptuales, teóricos y empíricos para el análisis de los formadores de formadores. Lo que busca es, por un lado, contribuir al estudio de la formación permanente con indicadores que permitan conocer las características personales y profesionales de los FF que trabajan en los PP y, por otro, establecer conexiones entre tales características, considerando como factor mediador el género de estos actores.

\section{MARCO CONCEPTUAL}

A continuación se explicita la teoría del objeto de estudio. Para ello se presenta la discusión bibliográfica referida a género, formación de profesores y perfil profesional. Además, se incluyen las características distintivas de los PP.

1.1. Género y educación. El concepto de género surge desde los conflictos que provocó el enfoque Mujer y Desarrollo para definir las estrategias orientadas a mejorar la posición social y económica de las mujeres (Cooks 2002). Producto de lo antes señalado, surgió la necesidad de superar las nociones esencialistas y universalistas y es así como se genera el levantamiento de la categoría "género". Se argumenta que el entendimiento de ser hombre o mujer tiene relación con un aprendizaje sociocultural más que con las características biológicas. El sexo se hereda y el género se adquiere a través de un aprendizaje, de aquí se desprende que, por un lado, la posición de la mujer no está determinada biológicamente sino culturalmente y, por otro, el rol central de la escuela en tal proceso (Abramo 2006; Arcos et al. 2007).

De lo anterior, se asume que el concepto de género es más que un constructo relacional. Según las Naciones Unidas (2001), es una teoría amplia que abarca categorías, hipótesis, interpretaciones y conocimientos relativos al conjunto de fenómenos históricos construidos en torno al sexo. El género como construcción simbólica capta los atributos asignados a las personas a partir de su sexo. Se trata de atributos biosicosociales que influyen sobre la organización de la sociedad en todas sus vertientes y sirven como base para las jerarquías del poder.

Entre los fines de la perspectiva de género está la participación en la construcción de una nueva configuración de la historia, la sociedad, la cultura y la política, desde las mujeres y con las profesionales (Cardaci 1998). Desde luego, revelar esta configuración de la realidad obliga a plantear nuevas alternativas de cambio social y a incluir la perspectiva de género en los procesos de producción científica asociados a las ciencias sociales, tanto en su proceso de configuración identitaria o perfil profesional como en la difusión académica. 
En la literatura internacional se constata cómo los avances en materia educativa no tienen su correlato en equidad de género respecto a inserción laboral, niveles de ingreso y participación en los procesos de adopción de decisiones y de poder (Bourdeau 1967; UNESCO 2006). Como consecuencia, los países han asumido el desafío de formular una tercera generación de reformas donde se sitúe la equidad de género en el centro de las políticas educativas. La preocupación en Chile, respecto a la equidad de género en el contexto educativo, se focaliza en los niveles de aprendizaje de ambos géneros. Un hallazgo importante es que, comparados con las mujeres, los hombres sienten más presión de sus padres para obtener un buen rendimiento y perciben un mayor apoyo para estudiar en el hogar (Andrade y Miranda 2001).

Sobre lo anterior, es válido preguntarse si en las familias chilenas la educación de los varones cobra prioridad por sobre la educación de las mujeres y, en el caso del FF, cómo la formación permanente refleja el éxito o fracaso de tales políticas. De ser esta última la respuesta, tal situación se constituye en uno de los principales desafíos para el principio de equidad que debería guiar las reformas socioeducativas actuales y futuras.

1.2. Formación permanente de profesores y perfil profesional. Para avanzar en la fundamentación de este trabajo es necesario detenerse a responder cómo se conceptualiza la formación permanente de profesores de primaria, qué modelos en uso presentan los autores abocados a su estudio $\mathrm{y}$, en el caso del formador de profesores, qué se entiende por perfil profesional.

En los estudios sobre los procesos de aprender y enseñar se constata que el constructo de formación permanente es polisémico (Jelbes 2008; Villagran 2008; Quintero 2008). En palabras de Alvarado (2006) el concepto (formación permanente) suele considerarse como sinónimo de formación en servicio, perfeccionamiento, reciclaje, entre otros. El uso de un término u otro se condiciona al origen político-ideológico del contexto en el cual se utiliza.

Según Avalos (2008) el concepto de formación permanente se sitúa bajo dos enfoques epistémicos que condicionan la gestión de los procesos formativos asociados a los docentes. Por un lado, el enfoque del Déficit y, por otro, de Desarrollo Profesional Docente. El primero enfatiza una visión sobre el docente que adolece de ciertas competencias, al cual hay que capacitarlo para su desempeño. El segundo orienta hacia una visión de progreso, donde los educadores son considerados portadores de saberes y experiencias previas y en continuo aprendizaje profesional. Mientras la perspectiva del Déficit pone su mirada en la incompetencia del sujeto en formación, en sus falencias e incapacidades, la segunda parte valorando las capacidades y destrezas que posee el sujeto para ayudarle en el fortalecimiento o desarrollo de estas (capacidades-destrezas). Para efectos de este estudio se asume la perspectiva del Desarrollo por valorar al sujeto como un ser en construcción. Lo anterior encuentra apoyo en la idea de Huberman (2004, en Rivera 2008) que entiende la formación permanente como la oportunidad de aprendizaje durante toda la vida.

Continuando con la idea anterior, Miranda et al. (2007) al tratar de responder a la inquietud sobre qué es la formación permanente, señalan que, en general, se asume como un proceso de formación profesional, que dura toda la vida y se inicia al momento en que el sujeto se plantea elegir la profesión docente. En esta idea se constata el reto que significa para el FF la tarea de apoyar la formación de tales profesionales, en la idea 
de ir configurando un perfil profesional lo suficientemente sólido y flexible que dote de identidad a este actor social.

En tal sentido, en este estudio se asume que el FF es el profesional que, siendo parte de un colectivo, media en la construcción de conocimientos con otros actores para -en conjunto- aprender y comprender conceptos y prácticas propias del ejercicio profesional docente. Entra en esta definición todo aquel que socializa un saber o contribuye para implementar proyectos educativos desde las diversas áreas del conocimiento. Además, son considerados formadores los especialistas que planean e implementan actividades, proyectos o programas de una determinada política educacional, tales como los gestores de los PP (González y Trebilcock 2007).

El perfil del docente y en especial del FF es variado, cada país posee una idea de perfil, que responde a sus políticas, necesidades, planes a implementar (Tabares 2005). En tal sentido, se asume con Hawes y Corvalán (2005) por perfil profesional un conjunto de rasgos y capacidades que, certificadas apropiadamente, permite que alguien sea reconocido por la sociedad como tal profesional, pudiéndosele encomendar tareas para las que se supone competente. Este perfil responde, en el caso de esta investigación, a un conjunto de rasgos personales y profesionales identificadores de competencia académica para acometer acciones en los programas de Postítulo.

1.3. Antecedentes de los Programas de Postítulo (PP). Los PP surgen luego de detectar la necesidad de actualizar los conocimientos disciplinares de los docentes de Enseñanza General Básica. Arellano y Cerda (2006) refuerzan esta idea, declarando que la mayoría de los docentes que ejercen en el $2^{\circ}$ ciclo de Educación Básica, en el Sector Subvencionado, tiene una formación inicial generalista, que resulta insuficiente respecto de las demandas que plantea el currículum de este nivel y ciclo educativo. Así, la argumentación básica es que los profesores de primaria no cuentan con la formación adecuada para desarrollar las demandas del actual currículum, entre cuyas razones se encuentran el carácter amplio de su tarea curricular, la carencia sistémica para desarrollar procesos de formación autodidacta y que el horario escolar es distribuido sin considerar recursos técnicos especializados.

Estas propuestas pretenden consolidar una alianza estratégica entre el Ministerio de Educación y las universidades chilenas que forman profesores. Esto surge en los 90 con los primeros programas de formación permanente, tales como el Programa de Perfeccionamiento Fundamental (PPF) y más recientemente con los de Apropiación Curricular (PAC). En tal sentido, según Beca (2006) es una alianza que dinamiza el sistema educativo y la formación de profesores.

Los objetivos del PP son mejorar el manejo del conocimiento del contenido disciplinar, fortalecer la autoestima y la profesionalidad de los docentes, mediante la especialización en áreas del currículum escolar y el posicionamiento de éste, ante los programas de estudio diseñados para segundo ciclo básico.

Metodológicamente, los PP se estructuran en dos modalidades. La primera es la de clases presenciales (700 horas). En ella los docentes desarrollan actividades de formación, con gran énfasis en la disciplina. En la segunda, denominada Seminario (175 horas) los profesores diseñan propuestas de innovación pedagógica. Además, se incorporan contenidos en género y ciudadanía.

Sobre los primeros, Fritz et al. (2008) señalan que su incorporación ha sido precaria e insuficiente En efecto, del total de propuestas estudiadas por estos autores $(n=174)$ 
menos de la mitad (44\%) incluye el enfoque de género. De aquellas que lo incorporan, un número importante lo hacen a nivel teórico y, en menor medida, como contenido curricular, quedando esto último al criterio del docente responsable del módulo.

Desde los resultados antes señalados, no se puede hacer una evaluación final de la eficacia de los PP, pero sí se destaca la preocupación por hacer de la formación permanente de profesores un objeto de estudio, pues tal como señala Jiménez (2008), los buenos resultados en las pruebas estandarizadas internas (Ej. SIMCE) se encuentran estrechamente vinculados a docentes que se forman continuamente.

\section{METODOLOGIA}

El presente estudio se inscribe dentro del paradigma cuantitativo, la investigación se adscribe a la característica de una investigación básica, el tipo de estudio es descriptivo, la técnica de recogida de datos utilizada es la historia de vida que será expresada en el instrumento Ficha Biográfica, aplicada a los FF, que se inserta en las propuestas oficiales de los PP. La técnica de análisis de los datos corresponde a las relacionadas con la estadística descriptiva, en la cual se buscaran medidas de tendencia central y contingencia.

El diseño del estudio es transversal y censal, dado que existe un corte en el tiempo, los PP se desarrollan desde el año 2004 a la fecha y el estudio se enfoca sólo en las propuestas del 2007; y descriptivo porque reconoce la existencia de estudios anteriores que exploran los PP como objeto de estudio (Miranda 2007; González y Trebilcock 2007; Rivera 2008).

2.1. Las Variables. Las variables responden al modelo de investigación evaluativa planteado por Stake (1985) y a la operacionalización del concepto perfil profesional descrito en el marco teórico. Estos dimensionan el constructo en tres factores del perfil, a saber: a) Personales. Para este estudio las variables de entrada son aquellas consideradas de orden personal u origen y se definen operacionalmente como edad, rango etario, género, título y grado académico; b) Laborales. Conocidas también como de proceso y se operacionalizan en programa, experiencia en aula, rango de experiencia, años de experiencia, categoría académica, dedicación horaria y cargo en el programa, y c) Académicas. Denominadas de salida o resultado y se definen operacionalmente en publicaciones según tema, actualidad, tipo, idioma y autoría.

2.2. Población. La población de esta investigación está constituida por 363 académicos que participaron en el desarrollo de los PP con mención para segundo ciclo básico, en 17 universidades del país. La distribución geográfica de los sujetos de estudio es de carácter nacional (Norte a Sur), esto implica que los FF responden a diversidad geográfica, cultural, social y étnica. Se estudiaron 37 propuestas, divididas en 4 grupos, según área curricular (PPMAT, PPCN, PPECS y PPLC).

2.3. Instrumento. La unidad de análisis del estudio estuvo constituida por los antecedentes curriculares de los FF incluidos en las propuestas. Para ello se confeccionó una Pauta de Ficha Biográfica, siguiendo el modelo aplicado por el CPEIP para la evaluación de los equipos de gestión que participan en el desarrollo de las propuestas presentadas a 
licitación, en las cuales el FF debe incorporar antecedentes de la carrera académica y de desempeño profesional. La Pauta consta de 32 ítemes relativos a los datos antes mencionados, distribuidos según factores a) personales, b) profesionales y, c) de productividad científica.

Con el fin de comprobar la validez del instrumento de análisis se sometió el instrumento a evaluación: del equipo de trabajo, triangulación con otros estudios y juicio de expertos. De tal forma, se califica el instrumento como adecuado para medir el Perfil Profesional docente de FF.

2.4. Análisis de los datos. Se llevó a cabo una serie de operaciones estadísticas descriptivas (cálculo de medias aritméticas, desviación estándar y construcción de tablas de contingencia y gráficos de dispersión), a fin de abordar de mejor manera la construcción del perfil de los docentes formadores de profesores que participan en las propuestas de los PP, esto mediante el uso del Programa Estadístico computacional SPSS, versión 11.5. Además, la depuración de los datos se realizó mediante el proceso de doble digitación. Finalmente, los resultados estadísticos fueron triangulados con el marco teórico de referencia y datos de estudios empíricos relativos a la eficacia de la formación permanente de profesores en general y de modo particular a los PP.

\section{RESULTADOS DEL ESTUDIO}

En este apartado se describen y analizan de manera sucinta los principales resultados derivados del estudio empírico de los datos, haciendo énfasis en los aspectos vinculados al género.

3.1. Género y perfil del formador de formadores. El considerar el género como un foco de análisis, adquiere pertinencia actual en el debate referido a la brecha entre la educación pública y la privada en el acceso a educación de calidad y a la educación superior. Además, adquiere relevancia económica por la introducción masiva de la mujer en el mercado laboral, siendo uno de ellos la academia. Se observa en distintas profesiones la desigualdad de oportunidades y el predominio del sexo masculino por sobre el género femenino, situación que se quiere revertir con distintas políticas y medidas elaboradas por el Gobierno.

Es necesario recordar que se investiga la población de formadores de formadores que laboran en 37 Programas de Postítulos, pertenecientes a 17 universidades del país, cuya edad promedio es de 49 años $^{2}$. A continuación la tabla 1 y gráfico 1 presentan los resultados del análisis estadístico de la distribución de los formadores según género.

2 Para un mayor detalle de las variables del perfil personal en Rivera (2008). 
Tabla 1

Distribución de formadores de Postítulo, según género

\begin{tabular}{|c|l|c|c|}
\hline \multicolumn{2}{|c|}{ Variable } & Frecuencia & Porcentaje \\
\hline \multirow{3}{*}{ Género } & Hombre & 213 & 58,7 \\
\cline { 2 - 4 } & Mujer & 150 & 41,3 \\
\cline { 2 - 4 } & Total & 363 & 100 \\
\hline
\end{tabular}

Fuente: Rivera, 2008.

La tabla 1 muestra que la población masculina se sobrepone con un $59 \%$ ante la femenina, existiendo una diferencia de $17 \%$ entre ambos grupos, donde 6 de cada 10 FF son hombres. La razón de hombre a mujer es de 1,42. Cifra que da cuenta de una tendencia que si bien evidencia superioridad numérica de varones, contrasta con los docentes de primaria del país, cuya proporción es inversa (Miranda 2005). Si se contrasta el número de profesores (363) con el número de docentes participantes en los PP (620) se puede constatar el carácter tutorial de los programas, es decir, la razón equivale a 1,7. Esto constata el carácter personalizado de las propuestas.

A continuación, en el grafico 1 se observa desagregadamente el género según mención del Postítulo, donde se manifiesta el proceder en la composición de los equipos de FF.

\section{Gráfico 1}

Distribución de género del Postítulo, según mención

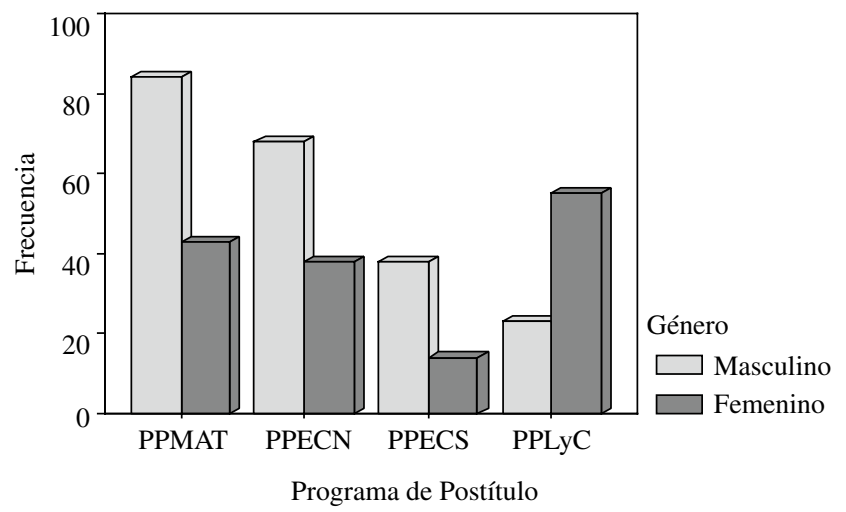

Fuente: Rivera, 2008.

La distribución de género por mención de los PP del gráfico 1 evidencia el predominio masculino en tres de los cuatro programas: PPMAT, PPECN y PPECS. La excepción es PPLC, donde se constata un predominio femenino (71\%), es decir, 7 de 
cada 10 formadores son este género. Tema que, según las tesis reproductivas, respondería a la asociación que se hace entre lo femenino y el cultivo de las humanidades (Bourdeau 1967; Beyer et al. 2002), situación que se reproduce en la educación primaria del país en el mayor logro escolar en Lenguaje y Comunicación por parte de las niñas y de los varones en Matemáticas y Ciencias en evaluaciones nacionales (Jiménez 2008) e internacionales (ILLECE 2008) para la educación primaria. Tal distribución de género en la conformación de los equipos docentes y su correlato en los resultados escolares es un dato que evidencia este estudio y que requiere ser atendido por los especialistas en el tema, situación que se prevé, no solo un aporte de esta investigación, sino también una línea de indagación a explorar en futuros análisis.

La tendencia del perfil laboral de los formadores de profesores es ostentar el título de Profesor de Educación Media (77\%), con grado de magíster en la mención del PP. La mayor parte de la población estudiada (77\%) reconoce tener experiencia sólo en aulas de instituciones de nivel superior, que en promedio es de 17 años. Su rol en los programas estudiados es ser profesor o encargado de un seminario (87\%) y la dedicación al programa es entre 15 a 149 horas, considerada baja al relacionarla con las 875 horas de duración de la propuesta.

El PPMAT, 25 docentes (20\%) declaran tener experiencia en la enseñanza media y superior. En este PP existe solo un caso de una docente formadora que declara tener experiencia previa en enseñanza básica, esto se transforma en relevante por ser este nivel escolar el marco contextual en el cual desarrollan su trabajo los profesores beneficiarios de los Postítulo. Esta última situación es, sin duda, un punto relevante a la hora de teorizar sobre las prácticas pedagógicas y la adecuación del conocimiento del contenido al conocimiento del contexto educativo (Huberman 2004). Sobre ello es pertinente preguntarse qué hipótesis del estudiante poseen los formadores de profesores al momento de plantear la necesaria transferencia al aula del contenido de los cursos de postítulo. $\mathrm{O}$ en otro sentido, qué elementos se consideran por parte de las instancias ministeriales al momento de adjudicar las propuestas. Sin duda, cuestionamientos que deberían ser considerados al momento de analizar la influencia de las características de los FF, el logro escolar y su impacto en el aprendizaje de los docentes.

El PPECN presenta una clara diferencia entre el grupo de hombres y mujeres, con experiencia solo en nivel superior. Los hombres presentan una diferencia de 55 sobre 25 mujeres que declaran tener solo experiencia en aulas de nivel superior. En relación a la experiencia en más de un aula, la distribución de docentes formadores es equitativa, 13 hombres y 13 mujeres. Cabe destacar que en esta distribución ciertos sujetos de análisis combinan la experiencia en aulas de nivel superior y básico que, en este último caso, son generalmente mujeres. La presencia mínima de la mujer en el espacio escolar primario es nuevamente un factor de análisis, no solo para profundizar cómo estas profesionales dieron el salto de un nivel a otro. Una explicación plausible se puede encontrar en la masiva incorporación de las prácticas profesionales como eje importante de la formación y reclutamiento de docentes. En efecto, siguiendo a Avalos (2008) un resultado de los proyectos de fortalecimiento de la profesión docente fue la incorporación de prácticas graduales en los currículum de formación docente, donde un número importante de profesores del sistema escolar asumieron labores de supervisión o colaboración en esta tarea.

El PPECS ratifica la tendencia hacia una diferencia entre hombres (32 Formadores) y mujeres (12 Formadoras) con experiencia solo en nivel superior, manteniendo una pro- 
pensión que muestra que los FF tienden a construir un carrera académica con experiencia exclusiva en educación terciaria, mientras las formadoras de profesores muestran la misma tendencia, pero matizada, que incluye experiencias en aulas en los tres niveles.

En el PPLC se revierte la tendencia de los PP anteriores desde una mirada de género. En este programa las mujeres (36) se ubican sobre el grupo de hombres (13) con experiencia solo en aulas de nivel superior. En relación con la experiencia en más de un nivel del sistema educativo, las mujeres (19) se destacan por sobre los hombres (10) en el caso de las letras. La relación entre niveles generalmente se presenta como nivel medio y nivel superior, pero las formadoras de profesores tienen una relación fuerte con nivel básico y experiencia en nivel superior.

En lo que sigue, las variables título y grado describen el perfil académico de los FF y que, desde la perspectiva de género, proporciona claves sobre las plausibles explicaciones a la diferencia encontrada en la distribución en los grupos estudiados.

Tabla 2

Contingencia entre título profesional, grado académico y género

\begin{tabular}{|c|l|c|c|c|}
\hline \multirow{2}{*}{\multicolumn{2}{|c|}{ Título Grado }} & \multicolumn{2}{|c|}{ Género } & \\
\cline { 3 - 5 } & Licenciatura & 1 & 0 & 1 \\
\hline \multirow{4}{*}{ Básica } & Masculino & Femenino & Total \\
\cline { 2 - 5 } & Postítulo & 0 & 1 & 1 \\
\cline { 2 - 5 } & Magíster & 6 & 7 & 13 \\
\cline { 2 - 5 } & Doctorado & 1 & 1 & 2 \\
\cline { 2 - 5 } & Total & 8 & 9 & 17 \\
\hline \multirow{4}{*}{ Media } & Licenciatura & 16 & 16 & 32 \\
\cline { 2 - 5 } & Postítulo & 3 & 11 & 14 \\
\cline { 2 - 5 } & Magíster & 71 & 55 & 126 \\
\cline { 2 - 5 } & Doctorado & 54 & 28 & 82 \\
\cline { 2 - 5 } & Total & 144 & 110 & 254 \\
\hline
\end{tabular}

Fuente: Elaboración propia.

La tabla 2 describe el perfil académico desde la interacción entre título profesional y grado académico en relación con el género. De ello se puede observar que la mayor parte de la población estudiada posee título de profesor de Enseñanza Media como formación inicial; la mayoría de los FF que alcanzan el grado doctoral son de género masculino (54) a diferencia con el género femenino que en su mayoría detenta el grado de Magíster, siendo menos las formadoras con doctorado (28). Los académicos que tienen por formación inicial la enseñanza General Básica (17) es equitativa entre hombres y mujeres, alcanzando en su mayoría el grado de Magíster, aunque se presentan dos casos con grado Doctoral, uno para cada género. 
Sin duda es importante constatar como el título profesional se relaciona con el grado académico y el género del FF. Al parecer, tal como lo evidencian estudios sobre la historia del sistema educativo chileno, persiste una tradición que vincula más la educación superior con la media que con la primaria (OCDE 2004). Desde la perspectiva del estudio (Cardaci 1998; PNUD 2005), esto es un elemento que ratifica la segmentación del sistema educativo chileno, donde la presencia de varones y mujeres en las instancias que jerárquicamente se estructuran siguiendo una lógica de "pirámide profesional", los varones tienen una mayor presencia en la cima (Coordinadores-Doctores) y las mujeres en la base (Docentes-Magister) de la jerarquía académica asociada a los PP.

A continuación la tabla 3 presenta los resultados del cruce de variables relativas a la productividad científica, el género y la mención del PP.

\section{Tabla 3}

Productividad científica del formador de profesores según mención y género

\begin{tabular}{|c|c|c|c|c|}
\hline \multirow{2}{*}{$\begin{array}{c}\text { Programa } \\
\text { de Postítulo }\end{array}$} & \multirow{2}{*}{ Tema de la Publicación } & \multicolumn{2}{|c|}{ Género } & \multirow[b]{2}{*}{ Total } \\
\hline & & Masculino & Femenino & \\
\hline \multirow{4}{*}{ PPMAT } & En el Área de la Mención & 32 & 17 & 49 \\
\hline & Área de Educación & 16 & 7 & 23 \\
\hline & Otras Áreas o Más de 1 & 8 & 3 & 11 \\
\hline & Total & 56 & 27 & 83 \\
\hline \multirow{5}{*}{ PPECN } & En el Área de la Mención & 41 & 17 & 58 \\
\hline & Área de Educación & 5 & 2 & 7 \\
\hline & Formación Permanente & 2 & 0 & 2 \\
\hline & Otras Áreas o Más de 1 & 4 & 2 & 6 \\
\hline & Total & 52 & 21 & 73 \\
\hline \multirow{4}{*}{ PPECS } & En el Área de la Mención & 19 & 6 & 25 \\
\hline & Área de Educación & 4 & 2 & 6 \\
\hline & Otras Áreas o Más de 1 & 5 & 0 & 5 \\
\hline & Total & 28 & 8 & 36 \\
\hline \multirow{6}{*}{ PPLC } & En el Área de la Mención & 12 & 23 & 35 \\
\hline & Área de Educación & 2 & 18 & 20 \\
\hline & Formación Permanente & 0 & 2 & 2 \\
\hline & Otras Áreas o Más de 1 & 2 & 3 & 5 \\
\hline & Total & 16 & 46 & 62 \\
\hline & Total general & 152 & 102 & 254 \\
\hline
\end{tabular}

Fuente: Elaboración propia. 
La tabla 3 permite observar el perfil académico según área de investigación, expresada en publicaciones de los FF. De lo anterior, se observa que los académicos más productivos son los adscritos al PPMAT (83), luego, PPECN (73) y PPLC (62) y, en menor medida, PPECS (36). Cada programa presenta publicaciones en las distintas categorías incluidas en el estudio, siendo la más recurrida las publicaciones en el área de la mención, las cuales responden al interés de cada Postítulo.

Desde la tabla 3 se evidencia que el género masculino presenta 152 publicaciones (60\%) y el femenino 102 (40\%). Es decir, en los FF hay una diferencia de 20 puntos porcentuales según género. Esto es consistente con el número de profesores varones y mujeres de la población del estudio. Por otro lado, no se observan diferencias según género en el tema de las publicaciones, presentándose en ambos géneros una mayor presencia de publicaciones en el área de la mención. No obstante a esta tendencia general, en los casos de los PPMAT y PPLC se constatan publicaciones en educación, siendo en el primero un tema abordado preferentemente por hombres y en el segundo por mujeres. Esto último puede ser una respuesta a la relevancia política y curricular que tienen estas áreas del saber en el sistema educativo chileno (Miranda 2007), expresada en su presencia en el currículum escolar y en los sistemas de evaluación estandarizados y, por otro, la presencia de líneas de investigación en la enseñanza de estas áreas en las facultades de Educación del país.

\section{DISCUSION Y CONCLUSIONES}

La discusión considera las características distintivas de los antecedentes personales, laborales y académicos de los FF que laboran en los PP, haciendo énfasis en la perspectiva de género y productividad científica, medida en publicaciones.

Este es un trabajo pionero, en Chile, que describe empíricamente la formación docente desde uno de los actores "ausentes" de la discusión sobre la calidad educativa: el FF. Por otra parte, se aporta al conocimiento sobre las características del FF según género y perfil profesional, operacionalizado en los equipos de trabajo que desarrollan los PP para docentes de segundo ciclo básico.

Los PP constituyen propuestas formativas de carácter "extensivo", cuya duración excede en gran medida a la mayoría de los programas de formación permanente que se desarrollan en el país, siendo solo comparables con un programa de magíster. En efecto, los 18 meses que dura el programa hacen de este perfeccionamiento una propuesta atípica que en términos económicos y de resultados es importante investigar. Es por ello que un número importante de investigaciones recientes han hecho de este programa un objeto de estudio (Cancino et al. 2005; González y Trebilcock 2007; Miranda 2007; Villagrán 2008; Jelbes 2008; Rivera 2008; Quintero 2008; Fritz et al. 2008). En tal sentido, su búsqueda por comprometer a los académicos de las Facultades de Educación y áreas de las menciones de los PP constituye una apuesta pertinente para generar sinergias y búsquedas compartidas (MINEDUC-Universidades) de mejoras y respuestas a las necesidades de formación permanente constatadas por la OCDE (2004) relativas a la inseguridad del manejo del conocimiento de los profesores. Este vínculo estratégico junto con tratar de responder a tales inseguridades debiera profundizar en la articulación de acciones que fortalezcan la carrera docente y que integren de manera simétrica a los mismos educadores, tal como 
se observó en la creación de los estándares del MBE. Por otro lado, en este trabajo se pudo constatar un enfoque personalizado en los PP al evidenciar una relación tutorial (1 formador por cada 3 profesores de primaria), estableciendo la modalidad de seminario como una apuesta metodológica pertinente a los PP. Esta relación es similar a la que se observa en los programas de postgrado en las áreas de ciencias biológicas y de la salud (Miranda et al. 2008). Estos dos factores introducen nuevos elementos en la discusión acerca de la naturaleza de los postgrados en Chile (Ministerio de Educación 2000).

El perfil de los FF fue un elemento central del estudio. Se evidencia una consistencia en los programas y menciones respecto al énfasis que éstas le otorgan en su foco disciplinar (Ej. Matemáticas) y las características profesionales de los docentes que laboran en ellos y el producto académico asociado (publicaciones). En tal sentido, este estudio interpreta que las menciones constituyen un indicador de mayor eficacia en la productividad científica que la cantidad de docentes que laboran en las propuestas. Lo anterior, no puede obviar algo revelador: la baja productividad general de los FF en revistas indexadas, menor a 1 en promedio para cada académico en toda su vida profesional (en promedio 17 años laborando en la educación superior). Un nudo crítico a ser discutido con investigación más amplia, pero que no deja de ser un elemento preocupante. Una explicación plausible a esta evidencia es que tal como lo constata el marco teórico son muy pocos los académicos que declaran vocación por la investigación científica en Chile (OCDE 2008).

Las características personales y profesionales de los actores que actúan en los procesos de formación permanente de profesores constituyen un elemento emergente en la experiencia investigativa chilena asociada a la formación de profesores. Si bien es un tema emergente en la literatura internacional sobre los procesos de aprender a enseñar eficaces (Miranda 2005; Rivera 2008), la investigación sistemática en programas de formación en especialidades curriculares ha sido poco abordada en el país. El examen de 37 PP de 17 universidades del país y sus respectivos focos disciplinares (Matemáticas, Lenguaje y Ciencias) mostró diferencias entre las menciones objeto del estudio en la productividad científica y constitución según género.

Datos de la investigación no profundizados aquí indican que los FF privilegian publicar en revistas con comité editorial o en resúmenes de congresos, aun cuando éstas no representen indicadores relevantes para la carrera académica o de incentivo económico. Estas observaciones permiten sugerir que la publicación en revistas con indexación SciELO e ISI son herramientas a potenciar en la producción científica asociada a procesos de formación docente en Chile. Este estudio avanza en esta dirección de manera exploratoria, quedando todo un campo de indagación a desarrollar, sobre todo, pertinente a la gran preocupación que muestran las instituciones de educación superior al momento de ser publicados rankings de calidad educativa.

Como todo trabajo académico exploratorio, se deja constancia de las limitaciones del presente estudio. Una línea poco abordada de este reporte es la necesaria profundización en los aspectos cualitativos de la labor de los FF, que responda a interrogantes tales como ¿qué motivaciones están a la base de la participación de FF en los PP?, ¿son los programas reflejo de sus intereses profesionales e indagativos?, ¿están estos programas al servicio de los profesionales de primaria?, ¿cumplen los programas sus expectativas de formación?, entre otras. Todas preguntas que deben ser profundizadas en futuras investigaciones que tengan como objeto estudiar la enseñanza eficaz.

En conclusión, este estudio sistematiza y abre espacios para nuevas indagaciones en torno al FF que labora en los programas de formación permanente en Chile y la relevancia 
que tiene este actor en el desarrollo profesional docente. La profundización en indicadores de eficacia de los programas de formación y su impacto en el aprendizaje profesional y escolar deben ser elementos centrales en nuevas líneas de investigación, en proyectos de intervención y planificación académica, tendientes a la formación disciplinar y generación de conocimiento científico que ilumine las prácticas educativas eficaces.

Se agradece especialmente la colaboración en la reflexión de este artículo al equipo de estudiantes y egresados del Programa de Magíster en Educación de la Universidad Austral de Chile que constituyen el equipo de investigadores de la línea de pesquisa "Eficacia de la formación permanente de profesores" Sres. Mauricio Jelbes, John Quintero, Erwin Muñoz y las Srtas: Pamela Muñoz, Silvia Salinas, Evelyn Mansilla y Patricia Villagrán, cuyo diálogo permanente ha sido fundamental para la concreción de este trabajo.

\section{BIBLIOGRAFIA}

Abramo, L. (2006). Trabajo decente y equidad género en América Latina. Santiago, Oficina Internacional del Trabajo, O.I.T.

Alvarado. L. (2006). Formación continua de profesores en servicio: formación de formadores. En Profissionais da Educação. Políticas, formação e pesquisa. Vol. III: 75-89.

Andrade, M. y Ch. Miranda (2001). Predicción del rendimiento académico lingüístico y lógico matemático por medio de las variables modificables de las inteligencias múltiples y del Hogar, Boletín de Investigación Educacional 16: 301-315. Santiago, Pontificia Universidad Católica de Chile.

Arellano, M. y A. Cerda (2006). Programa Postítulos de Mención para Profesores de Segundo Ciclo de Educación Básica, en Arellano, M. (eds.), Formación Continua de Docentes: un camino para compartir 2000-2005, CPEIP, Santiago de Chile: Maval Editores.

Arcos, E. et al. (2007). Perspectiva de género en la formación de profesionales de la salud: Una tarea pendiente, Rev. Méd. Chile 135: 708-717.

Avalos, B. (2008). Entrevista, inédita, en el marco del proyecto FONDECYT No 11060128.

Beca, C. (2006). Las políticas de formación continua de docentes: avances y desafíos, en Arellano, M. (eds.), Formación Continua de Docentes: un camino para compartir 2000-2005, CPEIP, Santiago de Chile: Maval Editores.

Bernasconi, B. y C. Rojas (2003). La educación Superior en Chile, Santiago, Banco Mundial.

Bourdeau, P. (1967). Los Herederos. Barcelona: Paidós.

Cancino, V. et al. (2005). Seguimiento del postítulo de mención en matemáticas para docentes de segundo ciclo de Enseñanza General Básica. Santiago: MINEDUC/CPEIP-OEI.

Cazden, C. (1991). El discurso en el aula. Barcelona: Paidós.

CINDA. (2002). Indicadores universitarios: Experiencias y desafíos internacionales de educación. Centro Universitario de Desarrollo, CINDA. Santiago de Chile: Ediciones Gestión Universitaria.

Cooks, L. (2002). Constructing gender pedagogies: Desire and resistance in the alternative classroom. Communication Education 53: 293-310.

Colegio de Profesores, A. G. (1997). Informe Final. Primer Congreso Nacional de Educación. Sin editorial. Santiago.

Figueroa, J. (2001). Los procesos educativos como recurso para cuestionar modelos hegemónicos masculinos, Diálogo y Debate de Cultura Política. Centro de Estudios para la Reforma del Estado 4 (15-16): 7-32.

Fritz, H. (coordinadora), J. Olavarría, T. Valdés, R. Ferrada, R. Molina y D. Da Silva (2008). Estudio acerca de la presencia de la perspectiva de género en el programa de formación para la apropiación curricular con apoyo de universidades y el programa de postítulos de mención. MINEDUC. Santiago. 
González, M. y M. Trebilcock (2007). Estudio de Seguimiento y Evaluación de los postítulos de mención para profesores de Segundo Ciclo de Educación Básica. Informe Final. Documento de trabajo preparado para CPEIP, MINEDUC.

ILLECE (2008). Laboratorio de Evaluación de la UNESCO. UNESCO, Madrid.

Jewkes, R. (2004). A prescription for gender, health and human rights. Lancet 363: 91.

Jiménez, M. (2008). Resultados del SIMCE. Discurso de la Ministra de Educación. En www. mineduc.cl/noticias.

Lamas, M. (2000). "La antropología feminista y la categoría de género", en Montecino, S. y C. Donoso, Teorías de género, Módulo I. Centro Interdisciplinario de Estudios de Género, Facultad de Ciencias Sociales, Universidad de Chile, pp. 57-84.

Lavados J. (2003). La educación superior chilena y los desafíos de la globalización. Disponible en: http://www.cse.cl/CSE/Asp/WEB_CSEpublic_pdf.asp

Lenn M. (2008). La Globalización de la educación superior: Temas Transnacionales de acceso, movilidad y calidad. Disponible en: http: www.cse.cl/doc/WEB_CSE public_peace.pdf

Loewenberg, D. (2008). Simons, J., H. Wu. The Final Report of the National Mathematics Advisory Panel U.S. Department of Education.

Magendzo, A. (2003). Formación ciudadana y objetivos transversales, en Hevia, R. (editor), La educación en Chile hoy. Santiago: Universidad Diego Portales, pp. 335-346.

Ministerio de Educación (2000). Normas de Procedimiento para la Evaluación Experimental de la Calidad de los Programas de Postgrado de las Universidades Autónomas. Decreto Exento $\mathrm{N}^{\circ} 3380$.

Miranda, Ch. (2005). Formación permanente de profesores. Impacto en sus competencias profesionales. Santiago: Pontificia Universidad Católica de Chile.

Miranda, Ch., P. Rivera, J. Quintero, M. Jelbes y P. Villagrán (2007). Concepciones del formador de profesores sobre la formación permanente. Revista Investigación Educativa. Santiago: PUC.

Miranda, Ch., H. Ibarra, E. Arcos y M. Concha (2008). Formación científica y ética en los PP de especialista en medicina en Chile. Revista Calidad de la Educación, pp. 123-142. Consejo Superior de Educación. Santiago.

Naciones Unidas, PNUD (2001). Informe sobre Desarrollo Humano 2001: Poner el adelanto tecnológico al servicio del desarrollo humano. Disponible en: http://www.undp.org/hdr2001/ spanish/spcptodo.pdf.

Núñez, I. (1999). La reforma educativa chilena. Cap. II. Los profesores. Madrid: Océano.

OCDE (2004). Informe del Comité Técnico sobre el sistema educativo chileno. MINEDUC. Santiago.

OCDE (2008). El Sistema de Educación Superior en Chile. MINEDUC. Santiago.

Rivera, P. (2008). Perfil profesional de los formadores de formadores del programa de Postítulo. Tesis de Postgrado, Valdivia, Universidad Austral de Chile. Valdivia

SIMCE (2007). Resultados del Sistema Nacional de Medición de la Calidad Educativa. En www. mineduc.cl/17 noviembre 2008 .

Stacki, S. y Monkman (2003). Change Through Empowerment Processes: women's stories from South Asia and Latin America, Compare 33: 173-189.

Stake en P. Cárdenas (2002). Investigación Evaluativa del Currículo de Formación Docente inicial. Temuco: Universidad Católica de Temuco.

Tarrés, M. (1998). ¿Importa el género en la política? En Género y cultura en América Latina, Cultura y participación política. Ed. El Colegio de México, pp. 13-32.

UNESCO (2006). Artículo 4. Fortalecimiento de la participación y promoción del acceso de las mujeres. Declaración mundial sobre la educación superior en el siglo XXI: visión y acción preámbulo. Aprobada por la Conferencia Mundial sobre la educación superior, realizada en Paris 1998. Disponible en: http://www.unesco.org/cpp/sp/declaraciones/ world.htm. Consultado el 18 de agosto. 